\title{
UNUSUAL AND LATE RECURRENCE IN OVARIAN ADULT GRANULOSA CELL TUMOUR: A CASE REPORT
}

\author{
Serajun Noor ${ }^{1}$ Jashim Uddin Ahmed ${ }^{2}$ Shahanara Chowdhury ${ }^{3}$ Zillur Rahman ${ }^{3}$
}

\begin{abstract}
Summary
Granulosa cell tumour of ovary are rare hormonally active neoplasm characterized by indolent course, local spread with a preponderance for extremely late recurrence and high survival rate. We report a 60 year old lady presented in 2009 with aggressive looking growth in vulva 16 years after her total abdominal hysterectomy with Bilateral salphingo-ophorectomy with infracolic omentectomy for suspected stage1 granulosa cell tumour of ovary, confirmed by histopathology. She remained disease free for 13 years and in 2005 underwent resection of retroperitoneal mass with multiple peritoneal implant, confirmed to be a granulosa cell tumour after biopsy. Despite six course adjuvent combination therapy with complete response she presented after 4 years in 2009 with a second recurrence in vulva. She had tumour reductive surgery followed by chemotherapy and hormonal therapy but the disease was progressive.In conclusion late recurrence and repeat recurrence is a hallmark for granulosa cell tumour of ovary, so we emphasis the need for long term follow up and consider the possibility of recurrence when presented with acute abdomen after initial surgery for granulose cell tumour. But there is no standered management protocol and we review this patients treatment in the context of current literature.
\end{abstract}

Key words

Granulosa cell tumour of ovary; late recurrence

\section{Introduction}

Adult granulosa cell tumour (GCTs) of the ovary are rare neoplasm with indolent course, local spread with apreponderance for extremely late recurrence and high survival rate [1]. They represent approximately $2-5 \%$ of all ovarian malignancies [2].

1. Associate Professor of Obstetrics \& Gynecology Chittagong Medical College, Chittagong

2. Professor of Surgery Chittagong Medical College, Chittagong

3. Professor of Obstetrics \& Gynecology Chittagong Medical College, Chittagong

4. Associate Professor of Pathology Chittagong Medical College, Chittagong

Correspondence: Dr Serajun Noor
They exhibit many morphological, biochemical and hormonal feature of normal proliferating preovulatory granulosa cell including expression of gene encoding FSH receptor, FSH binding, synthesis of oestrogen and inhibin.GCTs most commonly present during the perimanupausal or early postmanupausal period, with peak incidence occurring between ages of 50 and 54 years. Ovarian granulosa cell tumour very unusual in Childhood appearing as precocious puberty in the most prepubertal patients. Adult patients usually present with lump in lower abdomen (clinical/USG) with menstrual disturbance or postmenopausal bleeding. In contrast to ovarian tumour of epithelial origin GCTs are considered to be of low malignant potential and are characterized by their slow growth, indolent course with preponderance for local spread ,extremely late recurrence and high survival rate. Only a small percentage of tumour metastasise. Recurrence developed in up to $25 \%$ of patients many years (10 to 20years) after removal of original tumour [3]. Most $85-90 \%$ of the primary tumour are in stage 1 with substantial size $>8 \mathrm{~cm}, 40 \%$ incidence of endometrial hyperplasia and 5-13\% endometrial carcinoma [4]. Macroscopically the tumours are solid, soft or firm with or without cystic area, pale yellow in colour. Estradiol, total inhibin, follicle regulatory protein, mullerian inhibitory substances, serum antimullerian hormone are used as useful marker for GCTs activity [5]. GCTs disseminate via same route as epithelial carcinoma. Prognostic factors depends on stage, tumour size, tumour rupture, histology, mitotic activity, nuclear atypia, DNA aneuploidy, P53 expression, high $\mathrm{Ki}$ index. Advanced stage, size $>8 \mathrm{~cm}$, tumour rupture, insuler or diffuse pattern,mitotic index $>4 / 10$ HPF,nuclear atypia like large nuclear area with $\mathrm{S}$ phase fraction, DNA content heterogenecity, nuclear grooving,P53 overexpression 1 and high Ki67 index are correlated with adverse prognostic factor [6].

Surgery is the traditional treatment of choice like epithelial tumour. Staging system used for GCTs is that applied for epithelial ovarian carcinoma(FIGO Staging) and is mportant at the time of initial operation as there is chance of recurrence. 
As most of the patient(78-91\%) present with unilateral(2-8\%bilateral) stage I disease a unilateral salphingo-phorectomy should be preffered if young patients desiring to preserve fertility.It is imperative to do an endometrial biopsy to exclude endometrial hyperplasia.For patients whose fertility is not an issue and for post manupausal patients,advanced disease Total abdominal hysterectomy with bilateral salphingo-ophorectomy with removal of all visible disease should be performed [2]. If peritoneal washings are disease free, clinically and biopsy reveals stagel disease adjuvent therapy like chemotherapy is not defined. Management of advanced, recurrent, metastasis should involve aggressive debulking surgery followed by either adjuvent combination chemotherapy, radiotherapy, hormonal therapy like medroxyprogesterone acetate ,magestrol, tamoxifen, GnRH analogue and drug like aromatase inhibitor, tyrosine kinase inhibitor ,monoclonal antibody (bevacizumab) [7]. Although the overall response rate is high, the impact on disease free or overall survival is unknown.Due to their tendency to recur years after initial diagnosis, prolong surveillance is essential with following recommendation for follow up. Physical examination with recto-vaginal examination $1^{\text {st }}$ year every 3 month, $2^{\text {nd }}$ to $5^{\text {th }}$ year every 4 month and after $5^{\text {th }}$ year 6 monthly life long.

CT scan after completion of therapy as base line study or for unexplained abdominal symptom or hormonal changes. Chest X- ray annually and tumour marker as available[2].

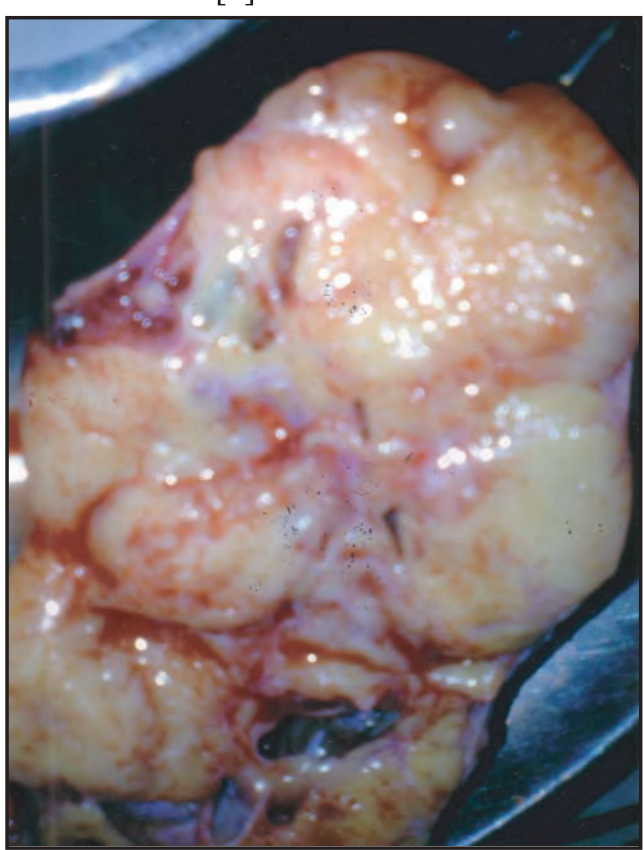

Fig 1 : Granulosa cell tumour on cut section

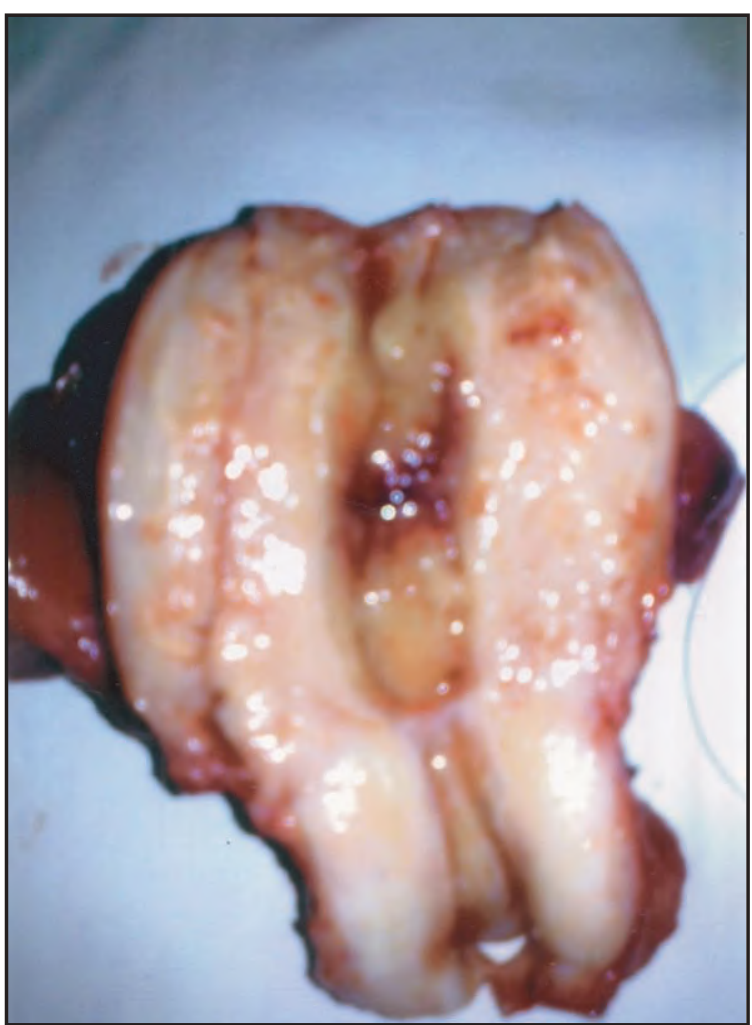

Fig 2 : Uterus on cut section showing endometrial hyperplasia

We report the case to have an awareness of recurrences, to emphasis the need for long term follow up in patients of granulosa cell tumour ,topredict the patients that are at risk of recurrence and to consider the possibility of recurrence when presented with acute abdomen after initial surgery for granulose cell tumour.

\section{Case report}

A 60 years old lady presented on 2009 with aggressive looking growth in vulva 16 years after her initial laparotomy when she was 44 years of age. Initially she had primary laparotomy followed by total abdominal hysterectomy ,bilateral salphingoophorectomy and infracolic omentectomy for a clinically suspicious stage1A granulosa cell tumourof ovary. Histology had confirmed type, assessed prognostic factor and stage of the disease. Considering the early stages of the disease and apparent complete surgical clearance she had not been offered adjuvant therapy. She had regular follow up for five years by physical and rectovaginal examination but base line CT scan,,USG were not done and Clinically there was no evidence of recurrence. She remained symptom free for 13 years after initial surgery in 1992 and admitted in department of surgery in the year 2005 with abdominal pain, generalized tenderness, significant rebound pain and muscle guarding. 
She developed unstable vital sign and then had immediate laperotomy. Macroscopic examination revealed a retroperitoneal mass with multiple peritoneal implant. The mass was resected with multiple biopsy. Histopathology confirmed the diagnosis as recurrent granulosa cell tumour. Patient was treated with six course of postoperative adjuvant chemotherapy with CAP regimen. Cisplatin $50 \mathrm{mg} / \mathrm{m}^{2}$,adriamycin $50 \mathrm{mg} / \mathrm{m}^{2}$ and cyclophosphamide $500 \mathrm{mg} / \mathrm{m}^{2}$ intravenously every 3 weeks. She had complete response clinically and had symptom free regular follow up without evidence of recurrence for 4 years.In the year 2009 at 60 years of her age she presented with a second recurrence in vulva with an angry looking growth simulating sqamous cell carcinoma but biopsy was consistent with recurrent granulosa cell tumour.Her serum oestrogen and inhibin level were also high.Tumour reductive surgery with nine cycle adjuvant therapy(Carboplatin and Peclitaxel) was offered in india. She had poor response to therapy. The tumour was both estrogen $(75 \%)$ and progesterone $(90 \%)$ receptor positive. Accordinglyoral megestrol acetate $40 \mathrm{mg}$ four times daily and injection GnRH analogue (leoprolide) was given but had progressive disease. She was advised for multiple chemotherapy regimens including bleomycin,etoposide,cisplatin for six cycle, oral etoposide for two cycle ,doxorubicin for nine cycle, gemcitabine for nine cycle. She received BEP therapy for four cycle and then denied further treatment and lost from follow up.

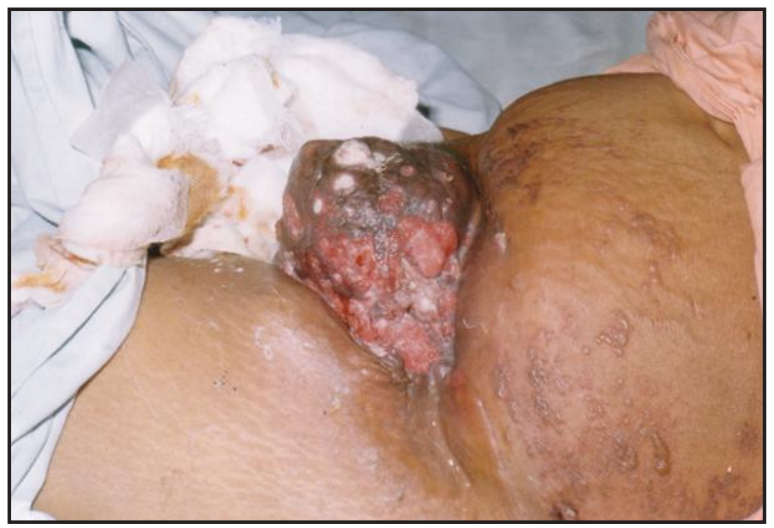

Fig 3 : Second recurrence on mons pubis

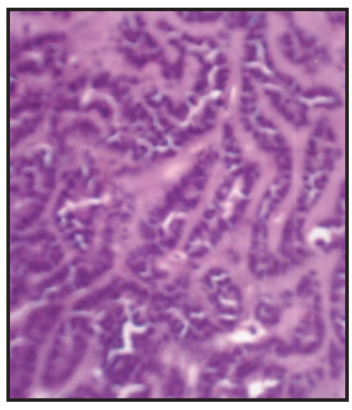

Fig 4 : Histology of GCT showing cords

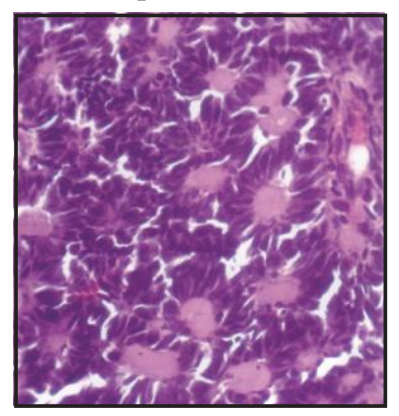

Fig 5 : Histology of GCT showing Call - Exner Body

\section{Discussion}

GCTs of ovary is a very uncommon ovarian sex cord stromal tumour of $5^{\text {th }}$ to $6^{\text {th }}$ decades but age of presentation can span from first to tenth decades [8]. Surgery represent the primary mode of treatment for early stage disease. Advanced or recurrent disease was found to be effectively treated with chemotherapy.However the recommendation have been made according to the findings of case series with level III evidence. Interval debulking can be considered in patients with gross residual disease and repeat cytoreduction was found to be useful for repeat recurrence.It appears that late stage of the disease and presence of gross macroscopic disease after primary has the most unfavourable effect on prognosis. The patient had regular clinical follow up for five years and disease free and symptom free.

She presented 13 years after primary surgery with acute abdomen as a symptom of recurrence and having complete response with tumour reductive surgery and combination chemotherapy for four years again represented with massive growth over mons pubis extending towards lower abdomen as second recurrence. There had been few cases with very late and repeat recurrences. There is also record of nearly 30 years of treatment for repeated and recurrent granulosa cell tumour of ovary.

Table I : Indolent nature of disease recurrent

\begin{tabular}{|c|c|c|c|}
\hline Author & $\begin{array}{l}\text { Number of } \\
\text { patients with } \\
\text { recurrent } \\
\text { disease }\end{array}$ & $\begin{array}{l}\text { Duration } \\
\text { between initial } \\
\text { diagnosis and } \\
\text { first recurrence }\end{array}$ & $\begin{array}{l}\text { Special feature of } \\
\text { recurrent disease }\end{array}$ \\
\hline Hasiakos et al [9] & 1 & 25 years & Presented as pelvic mass \\
\hline Givalos et al [10] & 1 & 10 years & $\begin{array}{l}\text { Sequential recurrences } \\
\text { presented as } \\
\text { Haemoperitoneum and as } \\
\text { a Hepatic mass respectively }\end{array}$ \\
\hline Auranen et a [11] & 7 & 2 to 12 years & $\begin{array}{l}\text { Presentation variable. } \\
\text { One patient had } 7 \text { Recurrences. }\end{array}$ \\
\hline Rha et al [12] & 11 & $\begin{array}{l}4 \text { month to } \\
18 \text { yrs. }\end{array}$ & $\begin{array}{l}\text { Variable presentation with } \\
\text { pelvic, Extrapelvic and para } \\
\text {-aortic recurrence }\end{array}$ \\
\hline \multicolumn{2}{|c|}{ Deanna teoh et al [13] 1} & 12 years & $\begin{array}{l}\text { Repeated recurrence for } \\
30 \text { years in Vagina,small bowel, } \\
\text { liver mass, diaphragm,lungs. }\end{array}$ \\
\hline
\end{tabular}

It is due to indolent nature of the disease and it is found to have a median time of six years. Due to potential for very late recurrence, some clinician prefer surveillance of upto 10 years [8]. 
Although the exact surveillance is still debateable pelvirectal examination along with CT scan and inhibin measurement ( if possible) is advocated by most. In our patient first recurrence with retroperitoneal mass can be diagnosed earlier by CT scan if the patient was aware of the abdominal recurrence. Serial measurement of inhibin may be helpful in follow up these women particularly in post manupausal age group [14]. The level of oestrogen and inhibin only measured during second recurrence and was found to be persistently high.

Platinum and taxane based chemotherapy have become the standered adjuvant treatment for ovarian cancer including GCTs [13]. Patient was treated with six course of CAP regimn and had complete response for four years.Gershenson et al reported an overall response of $63 \%$ in patients with CAP therapy,38\% complete response and $25 \%$ partial reponse [13]. Although data regarding the efficacy of carboplatin and paclitaxel in the treatment of GCTs is lacking multiple small studies demonstrated tumour response to platinum based regimen. One patients with repeated recurrence in 30 years of treatment was completely disease free for five month after having nine course of carboplatin and peclitaxel [13]. In a prospective study at M.D Anderson cancer centre from 1988 to $199351 \%$ patient treated with first line BEP therapy for recurrent disease remained disease free for three Years [13].

Hormonal therapy is another option for treatment for GCTs [15]. Though the patient had progressive disease with megestrol acetate and Leoprplide, there have been several reported cases of response toGnRH analogue in GCT. In a study of five patients with recurrent GCT had stabilization of disease or decreased inhibin level in first three months of treatment with GnRH agonist. A prospective study of Leuprolide acetate therapy for six patients with recurrent GCT shown $40 \%$ partial response rate with stable disease.

In the remaining $60 \%$.the progression free interval was 3-13 month [16]. The patient did not received radiotherapy but it has been used in recurrent disease. Though no randomized trial exist for defining the role of radiotherapy in the treatment of GCTs, yet with post operative radiotherapy is associated with improved survival. Sarvage et al treated 62 patients with GCTs, 8 of them received radiotherapy for advanced inoperable disease. Radiotherapy produced a number of long term remssion with an overall.

Response rate $50 \%$, with 3 remaining disease free for at least four years [17].
So ovarian AGCTs is a disease that tends to run an indolently malignant course and recurrence following initial therapy commonly occuring after more than 5 years, not infrequently after more than 10 years, sometimes after 20 years, very occassionally after more than 25 years. Therefore even after a disease free interval of 20 years it is not possible to reassure the patients with absolute dogmatism that she is cured with no chance of reccurrence. Neverthless most reccurence occur 5-10 years following the initial therapy and the role of defining the pathologic prognostic factor is to identify the patient at risk of recurrence [2].

\section{Conclusion}

Recurrent tumour can present with unexpected symptom. So it is imperative to recommend vigilant follow up protocol to diagnose early recurrence and thereby to achieve complete cure. However, the costeffectiveness of such a protocol is dubious. Selecting high risk women for prolong follow up is plausible solution and it may be worthwhile to produce clear guideline and possibly a scoring system to identify such high risk women who need prolong care after primary surgery.

\section{Disclosure}

All the authors declared no competing interestes.

\section{References}

1. Schumer ST,Cannistra SA.Granulosa cell tumor of the ovary.J Clin Oncol 2003; 21:1180-1187.

2. D.Pectasides,E Pectasides,A Psyrri:-Tumour review,Granulosa Cell tumour of ovary- Cancer treatment review $2008 ; 34: 1-12$.

3. HinesJF,Khalifa MA, Moore JL, Fine KP,Lage JM,Barnes WA.Recurrent granulose cell tumour of ovary 37 years after initial diagnosis: a case report and review of the literature. Gynaecol oncol 1996 ; $60: 484-488$.

4. Anikue C.Dawood MY.Karmen E.Granulosa cell tumour of ovary.Obset Gynaecol, 1998;51:214-220.

5. Katherine D,Crew,M,chil H. Cohen,Daniel H.Smith,Amy.D.Tiersten,Nikki M.Ferit, Dane L. Hershman Long natural history of recurrent granulosa cell tumour of ovary 23 years afterinitial diagnosis- A case Report and review of literature.Gynaecological oncology 2005;96:235-240.

6. WabersichJ,Fraces M. MazzanS.Marchettie M.Altavilla,G:The value of prognostic factors in ovarian granulosa cell tumours,Eur Gynaecol oncol 1998;19:69-72. 
7. Atula Kalurmachi,MS.FRCOG. Jeevan Prasona Mara singh MD.Late recurrence ovarian granulosa cell tumour- report on 2 cases.Annal academy of Medicine.October 2009; 138:125-131.

8. LeeYK.ParkNH,Kim JW,Song YS,KangSB, LeeHP.Characteristics of recurrence in adult-type granulose cell tumour.Int $\mathrm{J}$ Gynecol Cancer 2008;18:642-647.

9. HasiakosD,Papakonstantinou K, Karvouni E, Fotiou S.Recurrence of granulosa cell tumour 25 years after initial diagnosis.Report of a case and review of the literature.Eur J Gynaecol Oncol 2008;29:86-88.

10. GivalosN,IIakakos T,Machairas A,Gakiopoulou $\mathrm{H}$,Karatzas G Sequential recurrences G.Sequential recurrences of ovarian granulosa cell tumour 10 and 11 years after initial diagnosis as haemoperitoneum and subhepatic mass: A case report and review of literature.Eur J Gynaecol Oncol 2005;26:572-576.

11. Auranen A,SundstromJ,GrenmanS .Prognostic factors of ovarian granulose cell tumour:a study of 35 patients and review of the literature.Int $\mathrm{J}$ Gynecol Cancer 2007; 17:1011-1018.

12. Rha SE,Oh SN,Jung SE,LeeYJ, LeeAW, ByunJY.Recurrent ovarian granulose cell tumours:clinical and imaging feature.Abdo Imaging 2008;33:119-125.
13. Deanna Teoh, Ralph Freedman, Pamela T.Soliman :- Nearly 30 years of treatmet for Recurrent Granulosa cell tumour of the ovary:A Case Report and Review of the literature.Case Report oncol2010;3:14-18.

14. Start GC,Dawson L.M.Update on granulose cell tumour of ovary. Curr-opin obsted Gnecol2003;15:33-37

15. Hardy R D, Bell JG, Nicely CJ, Reid GC: Hormonal treatment of a recurrent Granulosa cell tumour of ovary: A report and review of the literature.Gynaecol Oncol 2005;96:865-869.

16. Fishman A,Kudelka AP,Tresukosol D,Edwards C L, Freed m an R S, K a plan A L, GirtnnerRE, KavanaghJj;Leoprolide acetate for treating refractory or persistent granulosa cell tumour.J REPROD AMAED 1996;41:393-396.

17. ChoanE,Samant R,Fung MFK,Le T,Hopkins L,Senterman M:Radiotherapy therapy for recurrent granulosa cell tumour of the ovary: a report on 3 cases with radiological evidence of response. Gynaecol Oncol 2006;102:406-410. 